\title{
Polymeric Flavonoids Obtained by Cross-Linking
} Reaction ${ }^{\dagger}$

\author{
Malgorzata Latos-Brozio * and Anna Masek \\ Institute of Polymer and Dye Technology, Faculty of Chemistry, Lodz University of Technology, \\ Stefanowskiego 12/16, 90-924 Lodz, Poland; anna.masek@p.lodz.pl \\ * Correspondence: malgorzata.latos@p.lodz.pl \\ + Presented at the First International Conference on “Green” Polymer Materials 2020, 5-25 November 2020; \\ Available online: https://cgpm2020.sciforum.net/.
}

Published: 4 November 2020

\begin{abstract}
Plant polyphenols are becoming more and more popular due to their strong anti-aging properties. The best researched and largest group of polyphenols are flavonoids. Flavonoids have high antioxidant and pharmacological activities and these properties are closely related to their structure. Certain structural elements of these compounds condition their properties and improve or degrade the activities. As a result of the polymerization of flavonoids, macromolecular compounds showing more favorable properties, such as, for example, bactericidal and antioxidant activity, can be obtained. The aim of this study is to polymerize selected flavonoids (quercetin and rutin) in reaction with a cross-linking compound. Glycerol diglycdyl ether (GDE) causes the crosslinking of quercetin or rutin monomers and the formation of polymeric structures. The study analysed the thermal stability of monomeric and polymeric flavonoids and their antioxidant activity. Poly(flavonoids) showed greater resistance to oxidation than their monomeric forms. Moreover, poly(quercetin) and poly(rutin) have a greater ability to reduce transition metal ions. Polymeric forms of quercetin and rutin can potentially be effective stabilizers, e.g., for polymeric materials.
\end{abstract}

Keywords: quercetin; rutin; polymerization; cross-linking compound

\section{Introduction}

Quercetin and rutin are popular flavonoids in the diet, arousing great interest due to their beneficial pharmacological properties and strong antioxidant properties. Quercetin is a flavonol occurring in fruit and vegetables. In food products, this flavonoid may appear in the form of

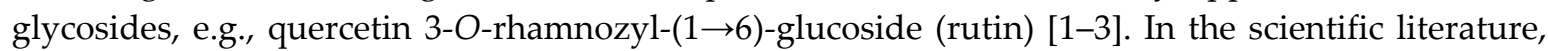
special attention is paid to the pro-health and antioxidant properties of both quercetin and its glycoside-rutin. The properties of flavonoids strictly depend on their chemical structure and may be modified, for example, by polymerization [4-6].

One of the methods of flavonoids polymerization is reaction with a cross-linking compound [7,8]. Sahiner proposed a one-stage microemulsion/crosslinking polymerization of quercetin [7] and rutin [8] using L- $\alpha$ lecithin as surfactant, cyclohexane as organic phase and glycerol diglycdyl ether (GDE) as a crosslinking agent. The obtained poly(quercetin) was characterized by higher thermal stability, higher antioxidant activity and better antibacterial properties than the momomeric form [7]. Poly(rutin) also showed higher thermal stability than rutin. In addition, it was characterized by higher fluorescence than the monomer [8].

The aim of the research is to analyze the properties of polymeric forms of quercetin and rutin. Poly(querectin) and poly(rutin) were polymerized with the crosslinker according to the method described by Sahiner. This manuscript extends and complements Sahiner's analysis of 
poly(flavonoids). In the publications $[7,8]$, the author did not analyze the thermal changes of polymeric quercein and rutin using differential scanning calorimetry and did not study the ability of polymeric flavonoids to reduce multivalent metal ions-iron and copper. The ability of poly(flavonoids) to reduce transition metal ions is important as part of the evaluation of these compounds for use as a stabilizing agent which reduces the metals catalyze the aging process.

\section{Experiments}

\subsection{Polymerization}

Flavonoids polymerization was performed according to the method proposed by Sahiner $[7,8]$ with minor modifications.

\subsection{Differential Scanning Calorimetry DSC}

The prepared poly (flavonoids) was subjected to differential scanning calorimetry DSC. The samples were heated from -80 to $400{ }^{\circ} \mathrm{C}$ at a rate of $10^{\circ} \mathrm{C} / \mathrm{min}$ in an air atmosphere. For comparison, DSC of the reference quercetin and rutin was performed.

\subsection{FRAP and CUPRAC Analysis}

The FRAP (Ferric Reducing Antioxidant Power) and CUPRAC (Cupric Reducing Antioxidant Capacity) methods are used to assess the ability of compounds to reduce transition metal ions. The FRAP test is based on the reduction of the ferric ion $\left(\mathrm{Fe}^{3+} \rightarrow \mathrm{Fe}^{2+}\right)$ under acidic conditions. The CUPRAC assey is analogous to the FRAP method and consists of the reduction of $\mathrm{Cu}^{2+}$ to $\mathrm{Cu}^{1+}$. (1):

The ferric (FRAP) and cupric (CUPRAC) ions reducing capacity was according to the Formula

$$
\Delta \mathrm{A}=\mathrm{A}_{\mathrm{AR}}-\mathrm{A}_{0}
$$

where: $\mathrm{A}_{0}-$ absorbance of the reagent test, $\mathrm{A}_{\mathrm{AR}}-$ absorbance after reaction.

Absorbance measurements in the FRAP and CUPRAC tests were performed using a UVspectrophotometer (Evolution 220, Thermo Fisher Scientific, Waltham, MA, USA). A detailed description of the determinations the authors presented in the following publications $[9,10]$.

\section{Results and Discussion}

The research began with the thermal analysis of monomeric and polymeric flavonoid forms. Figure 1 shows DSC thermograms, and Table 1 summarizes the results of DSC analysis of the tested flavonoids and poly(flavonoids). Polymeric quercetin and rutin obtained by polymerization with a crosslinking compound, were characterized by a lower melting temperature $\mathrm{T}_{\mathrm{m}}$ than the melting temperature of reference polyphenols. The addition of a GDE cross-linker can lower the $\mathrm{T}_{\mathrm{m}}$ of the poly(flavonoids). Interesting results were obtained for the changes in the oxidation temperature $\mathrm{T}_{\mathrm{o}}$. Poly(quercetin) has a higher final oxidation temperature $T_{0}$ (by $39.2^{\circ} \mathrm{C}$ ) and a higher enthalpy of oxidation $\Delta \mathrm{H}_{\mathrm{o}}$ (about 6 times) then monomeric quercetin. Thus, the polymeric form of quercetin showed greater resistance to oxidation. The polymeric form of quercetin glycoside-poly(rutin) was characterized by a higher enthalpy of oxidation (about 15 times), but the oxidation temperature $\mathrm{T}_{\mathrm{o}}$ was lower than temperature of rutin by $58.8^{\circ} \mathrm{C}$. 

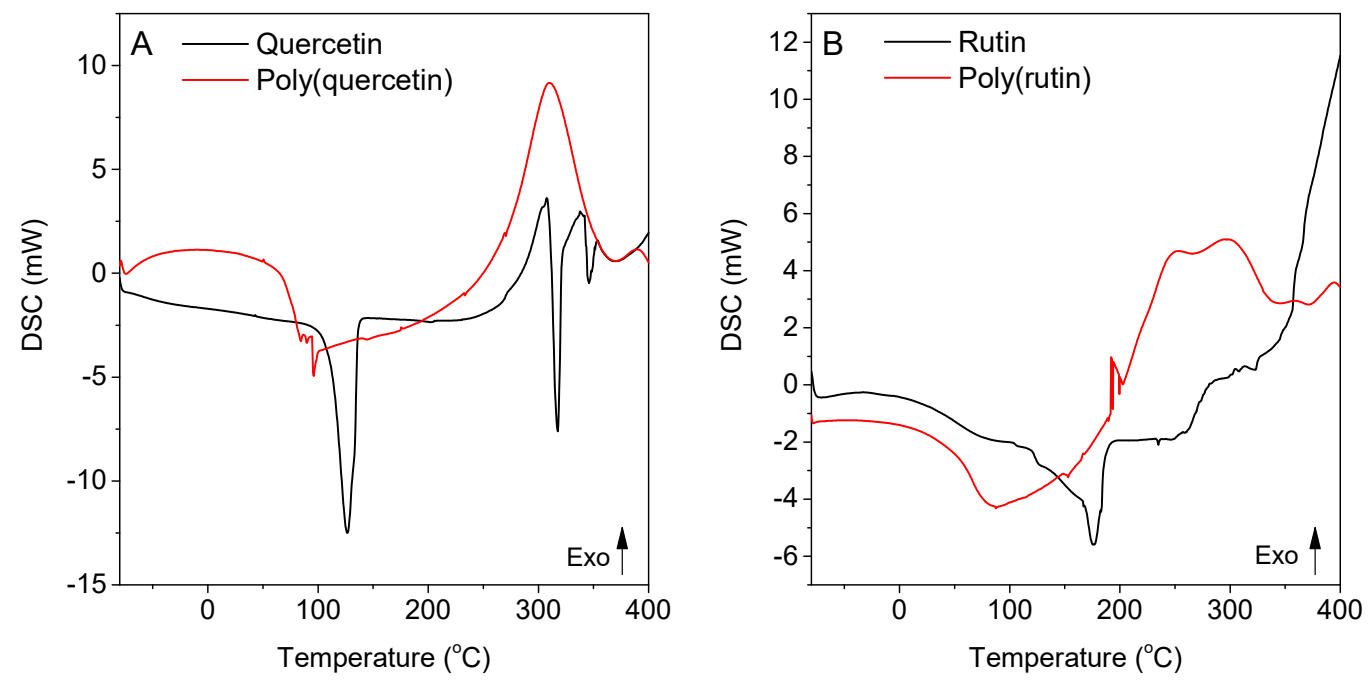

Figure 1. DSC thermograms of quercetin and poly(quercetin) (A) and rutin and poly(rutin) (B).

Table 1. DSC analysis of quercetin and poly(quercetin) (A) and rutin and poly(rutin) (B).

\begin{tabular}{lllll}
\hline Sample & $\Delta \mathbf{H}_{\mathrm{m}}[\mathrm{J} / \mathrm{g}]$ & $\mathrm{T}_{\mathrm{m}}\left[{ }^{\circ} \mathrm{C}\right]$ & $\Delta \mathbf{H}_{\mathrm{o}}[\mathrm{J} / \mathrm{g}]$ & $\mathrm{T}_{\mathbf{o}}\left[{ }^{\circ} \mathrm{C}\right]$ \\
\hline Quercetin & 193.0 & 111.0 & 99.9 & 312.4 (endset) \\
Poly(quercetin) & 119.2 & 48.6 & $\mathbf{6 2 6 . 5}$ & $\mathbf{3 5 1 . 6}$ (endset) \\
Rutin & 115.7 & 160.3 & 31.3 & 313.3 (endset) \\
Poly(rutin) & 118.3 & 52.4 & $\mathbf{4 6 0 . 4}$ & 258.5 (endset) \\
\hline
\end{tabular}

$\Delta \mathrm{H}_{\mathrm{m}}$ - enthalpy of melting, $\mathrm{T}_{\mathrm{m}}-$ melting temperature, $\Delta \mathrm{H}_{\mathrm{o}}$ - enthalpy of oxidation, $\mathrm{T}_{\mathrm{o}}$-oxidation temperature (final-endset).

The next step of the research was to determine the ability of flavonoids and poly(flavonoids) to reduce transition metal ions. Polyvalent metal ions can catalyze the aging processes of, eg. polymeric materials. Therefore, the ability to reduce and chelate such ions is a very important property that a stabilizer should fulfill. Figure 2 shows the ability of quercetin and poly(quercetin) (A) as well as rutin and poly(rutin) (B) to reduce iron (FRAP measurement) and cupric (CUPRAC assay) ions. The solutions of the reference quercetin and rutin flavonoids, at a concentration of $1 \mathrm{mg} / \mathrm{mL}$, showed a much lower ability to reduce iron and copper ions than solutions of poly(flavonoids). The poly(flavonoids) of quercetin and rutin, obtained as a result of the reaction with glycerol diglycdyl ether (GDE), were characterized by a better ability to reduce transition metal ions. The polymerization of these compounds resulted in a positive effect on the improvement of this property.
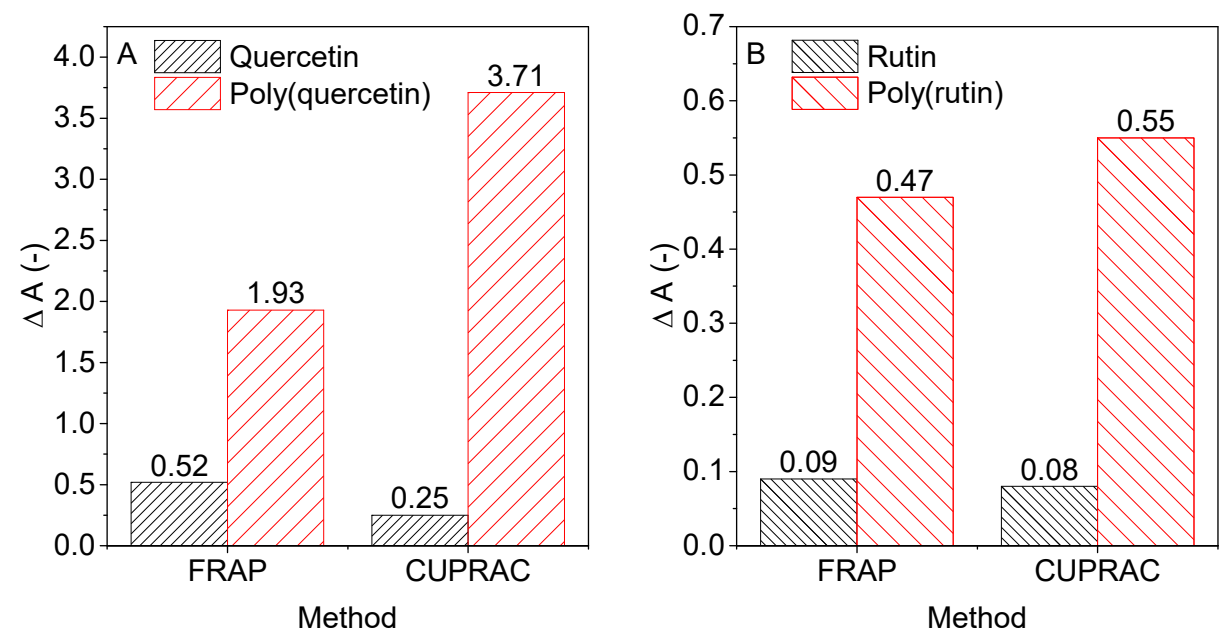
Figure 2. Ability of quercetin and poly(quercetin) (A) as well as rutin and poly(rutin) (B) to reduce iron and cupric ions measured by FRAP and CUPRAC methods.

\section{Conclusions}

Flavonoids polymerization with the cross-linking compound influenced their thermal and stabilizing properties. Poly(quercetin) was characterized by a higher oxidation temperature than its monomeric form. Poly(rutin) had a much higher enthalpy of oxidation than rutin. Moreover, both poly(flavonoids) showed a significantly higher ability to reduce multivalent metal ions that catalyze the aging processes. Due to their good resistance to oxidation and great ability to reduce metal ions, poly(quercetin) and poly(rutin) can potentially be good stabilizers, e.g., for polymeric materials and environmentally friendly materials.

Author Contributions: M.L.-B. and A.M. conceived and designed the experiments; M.L.-B. performed the experiments; M.L.-B. and A.M. analyzed the data; A.M. contributed reagents/materials/analysis tools; M.L.-B. wrote the paper. All authors have read and agreed to the published version of the manuscript.

Acknowledgments: This research was funded by the National Science Center, Poland (NCN), grant number 2018/31/N/ST8/02565.

Conflicts of Interest: The authors declare no conflict of interest.

\section{Abbreviations}

The following abbreviations are used in this manuscript:

$\begin{array}{ll}\text { DSC } & \text { Differential Scanning Calorimetry } \\ \text { CUPRAC } & \text { Cupric Reducing Antioxidant Capacity } \\ \text { FRAP } & \text { Ferric Reducing Antioxidant Power } \\ \text { GDE } & \text { glycerol diglycdyl ether }\end{array}$

\section{References}

1. Materska, M. Quercetin and its derivatives: Chemical structure and bioactivity-A review. Pol. J. Food Nutr. Sci. 2008, 58, 407-413.

2. Wang, W.; Sun, C.; Mao, L.; Ma, P.; Liu, F.; Yang, J.; Gao, Y. The biological activities, chemical stability, metabolism and delivery systems of quercetin: A review. Trends Food Sci. Technol. 2016, 56, 21-38.

3. Gullon, B.; Lú-Chau, T.A.; Moreira, M.T.; Lema, J.M.; Eibes, G. Rutin: A review on extraction, identification and purification methods,biological activities and approaches to enhance its bioavailability. Trends Food Sci. Technol. 2017, 67, 220-235.

4. Heim, K.E.; Tagliaferro, A.R.; Bobilya, D.J. Flavonoid antioxidants: Chemistry, metabolism and structureactivity relationships. J. Nutr. Biochem. 2002, 13, 572-584.

5. Wu, T.; Zang, X.; He, M.; Pan, S.; Xu, X. Structure-Activity Relationship of Flavonoids on Their AntiEscherichia coli Activity and Inhibition of DNA Gyrase. J. Agric. Food Chem. 2013, 61, 8185-8190.

6. Jacob, V.; Hagai, T.; Soliman, K. Structure-activity relationships of flawonoids. Curr. Org. Chem. 2011, 15, 2641-2657.

7. Sahiner, N. One step poly(quercetin) particle preparation as biocolloidand its characterization. Colloids Surf. A Physicochem. Eng. Asp. 2014, 452, 173-180.

8. Sahiner, N. One step poly(rutin) particle preparation as biocolloid and its characterization. Mater. Sci. Eng. C 2014, 44, 9-16.

9. Latos-Brozio, M.; Masek, A. Biodegradable Polyester Materials Containing Gallates. Polymers 2020, $12,677$. 
10. Masek, A.; Latos-Brozio, M.; Kałużna-Czaplińska, J.; Rosiak, A.; Chrześcijańska, E. Antioxidant Properties of Green Coffee Extract. Forests 2020, 11, 557.

Publisher's Note: MDPI stays neutral with regard to jurisdictional claims in published maps and institutional affiliations.

(c) 2020 by the authors. Submitted for possible open access publication under the terms and conditions of the Creative Commons Attribution (CC BY) license (http://creativecommons.org/licenses/by/4.0/). 